\title{
MEJORES PRÁCTICAS PARA UNA CADENA DE PRODUCCIÓN SOSTENIBLE
}

\author{
BEST PRACTICES FOR SUSTAINABLE PRODUCTION CHAIN \\ Elsa Esther Choy Zevallos* \\ Docente Asociada de la Facultad de Ciencias Contables
}

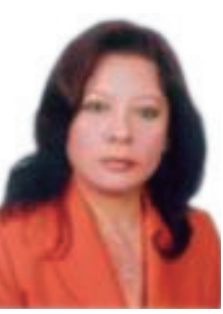

\author{
Universidad Nacional Mayor de San Marcos - UNMSM / Lima - Perú
}

[Recepción: Setiembre de 2015/ Conformidad: Octubre 2015]

\section{RESUMEN}

El trabajo es una continuación del tema: "Cadena de producción sostenible en costos y seguridad alimenticia nacional", que ha sido publicado en la Revista de Investigación Contable Quipukamayoc en la edición anterior. En esta oportunidad, se tiene como propósito desarrollar las mejores prácticas para lograr la mejora continua en la cadena productiva agrícola, que como se señaló anteriormente, garantice un desarrollo sostenible con viabilidad económica, ecológica, justicia social y humanística, donde se integren los procesos naturales con los de la producción, asi como mayor productividad de plantas y productos orgánicos.

El estudio desarrolla las mejores prácticas no contaminantes para una producción sostenible con la agricultura orgánica, tiene un enfoque metodológico deductivo de tipo descriptivo y presenta como resultado, una estructura de las mejores prácticas que agregan valor a la cadena productiva agrícola, bajo cinco dimensiones: social, ecológica, económica, cultural y responsabilidad social.

\section{Palabras clave:}

Producción agrícola orgánica; producción sostenible; mejores prácticas.

\begin{abstract}
The work is a continuation of the subject: "Chain of sustainable production costs and national food security “, which was published in the Journal of Accounting Research Quipukamayoc in the previous edition. This time, it is aimed to develop the best practices to achieve continual improvement in agricultural production chain, which, as noted above, ensures sustainable development with economic and ecological sustainability, social and humanistic justice, where natural processes are integrated with production as well as higher productivity of plants and organic products.

The study develops the best environmental friendly practices for sustainable production with organic agriculture, it has a deductive methodological approach of descriptive type, and presents and as a result, a structure of best practices that add value to agricultural production chain under five dimensions: social, ecological, economic, cultural and social responsibility.
\end{abstract}

\section{Keywords:}

Organic agricultural production; sustainable production; best practices.

\footnotetext{
* Doctora en Ciencias Contables y Empresariales, Magíster en Dirección Financiera y Contador Público Colegiado. Email: eschoyz@outlook.com
} 


\section{INTRODUCCIÓN}

Mientras la población mundial y nacional continúa creciendo, el sistema de producción agrícola moderno no ha superado sus prácticas contaminantes que afectan la fertilidad de la tierra, cantidad y calidad del agua y biodiversidad, impactando en los alimentos y; en consecuencia, la salud de la población.

Helfgott, S. y otros (2010) en la investigación referida a "Aptitud de tierras y oportunidades para el desarrollo rural en Perú", establece algunas cifras proyectadas para el Perú, donde estima que la población aumentará hasta 34 millones en el 2020 y 40 millones en el 2030, el aumento ocurrirá básicamente en los sectores urbanos, mientras que el número de personas en el sector rural será el mismo o disminuirá debido a las migraciones.

En tal sentido, los productores rurales tendrán que ser más eficientes para aumentar la producción de alimentos, esto será posible si se logra reorientar aquellas prácticas destructivas, en modelos de producción sostenible y consumo de alimentos sustentables, bajo un enfoque integrado y holístico que reúna aspectos sociales, ecológicos, económicos, culturales y de responsabilidad social, permitiendo agregar valor a la cadena de productos agrícolas orgánicos.

El presente trabajo recoge este planteamiento de mejores prácticas aplicada a los sistemas de producción orgánica, como una perspectiva de contribución hacia la cadena de valor sostenible, que estimule la economía local y la participación en mercados internacionales.

Para el cumplimiento del objetivo, se ha desarrollado el marco teórico relacionado con las buenas prácticas en agricultura, bajo cinco dimensiones planteadas por la Red de Acción de Agricultura Sostenible -SOAAN (2013). Asimismo, el estudio es relevante para los empresarios y comunidad académica, contribuyendo al conocimiento de las mejores prácticas en beneficio de nuestros recursos naturales.

\section{CADENA DE VALOR CON AGRICULTURA ORGÁNICA}

La agricultura orgánica es la clave para conservar y restablecer nuestros recursos naturales. La Federación Internacional de Movimientos Agrícolas Orgánicos (IFOAM), define que "La agricultura orgánica es un sistema de producción que mantiene la salud de las tierras, ecosistemas, y personas. Depende de procesos ecológicos, biodiversidad, y de ciclos adaptados a las condiciones locales, en vez de usar insumos con efectos adversos. La agricultura orgánica combina tradición, innovación, y ciencia que benefician el medio ambiente compartido y promueve relaciones justas y una buena calidad de vida para todos los involucrados."

\section{MEJORES PRÁCTICAS PARA UNA CADENA PRODUCTIVA SOSTENIBLE}

Como se señaló anteriormente, la Red de Acción de Agricultura Sostenible, establece ejemplos aplicativos de mejores prácticas para una producción orgánica sostenible, dividida en cinco dimensiones, de las cuales se cita algunas prácticas:

\section{Dimensión Social}

- Equidad, política y prácticas no-discriminatorias aplican para todas las partes interesadas. No hay sesgo de género en lo que respecta a empleos, remuneración, acceso a recursos y educación, ni a oportunidades laborales.

- Empleadores en todos los niveles de cadenas de abastecimiento, pagan salarios que son adecuados para mantener un estándar de vida decente y para el seguro social de todos los empleados.

- Toda tierra es usada bajo condiciones de libre y previo consentimiento de los habitantes originales.

- Las operaciones están claramente de acuerdo con los términos laborales y con todos los empleados.

- La labor forzada o involuntaria no es usada de ninguna manera en la cadena de valor. 
- Todo centro de trabajo provee a las personas con agua potable para beber, condiciones higiénicas de alimentación, e instalaciones sanitarias apropiadas.

- Los trabajadores son capacitados acerca de los riesgos inherentes a su entorno laboral. Se les ofrece protección adecuada contra el ruido, polvo, exposición solar, riesgos indebidos relacionados a maquinarias y equipos, y exposición a químicos y residuos.

\section{Dimensión Ecológica}

- Los operadores evalúan su uso del agua para determinar si sus prácticas depredan o degradan los recursos hidráulicos, y luego planean cómo mejorar su eficacia.

- El uso del agua es lo más eficiente posible mediante una minuciosa programación de uso, diseño de sistemas (que incluyen cosecha de agua y secuencias de cultivos), el uso apropiado de tecnología (que incluye irrigación y características de equipo de procesamiento), reduciendo pérdidas y fugas, y reciclándola a través del sistema cuando sea posible.

- Los consumidores de agua en cada localidad son conscientes de las necesidades de agua que otros consumidores tienen. El acceso a dicho recurso no se ve afectado de manera injusta.

- La extracción sostenible de agua dulce y su uso no perjudica el funcionamiento de los ciclos naturales del agua y ecosistemas.

- El suelo está protegido de pérdidas debido a la erosión y exposición casual o intencional a los elementos (sol, viento, agua, fuego, y tráfico de animales). Se mantiene cubierto con plantas y cubierta vegetal la mayor parte del tiempo.

- El contenido de la materia orgánica del suelo incrementa. Los agricultores mejoran la actividad biológica del suelo y son conscientes de las actividades que la afectan.

- Los agricultores toman en consideración las ventajas de incorporar abono compostado al suelo, tanto vegetal como animal, tales como la reducción de patógenos, prevención de filtración de nutrientes, y el fomento de nutrientes residuales en el suelo.

- Los agricultores dependen principalmente de prácticas culturales interrelacionadas que incluyen la rotación de cultivos, enemigos naturales, y manejo de la biodiversidad para controlar plagas, enfermedades, $y$ malas hierbas. Se prefieren materiales naturales y los menos tóxicos para la protección de los cultivos; se evitan los plaguicidas sintéticos y tóxicos.
- Todos los actores en la cadena de valor se preocupan de mantener y mejorar la biodiversidad. Los actores que no son productores principales se responsabilizan de sus propias actividades, además de los impactos de sus operaciones en los problemas de biodiversidad de los productores principales.

- El desarrollo sostenible no invade áreas de vegetación silvestre y otras áreas de alto valor de conservación, en particular bosques nativos, pendientes empinadas, vegetación ribereña, pantanos, manglares, y planicies aluviales.

- Los agricultores mantienen o restablecen vegetación natural alrededor de los arroyos, cauces naturales, pendientes empinadas y colinas, y otras áreas sensibles del ecosistema. Los pantanos naturales no deberán ser drenados.

- Los agricultores respetan el derecho de las especies existentes de mantener acceso a su hábitat natural para subsistir.

- Los agricultores no crían más animales de los que sus tierras puedan albergar. En caso de desvíos el agricultor toma en cuenta el impacto potencial a la contaminación ambiental, el uso de energía no-renovable, la emisión de gases con efecto invernadero, y el perfil nutricional de los productos animales.

- Los agricultores no permiten que los animales sobrecarguen los terrenos de pasto, lo cual podría conducir al agotamiento de nutrientes y a la pérdida de tierras por erosión, especialmente en áreas áridas o inclinadas.

- El uso de suministros de energía insostenible o el aumento de la huella de carbono es tomado en consideración en lo que respecta al transporte de abono, alimento para el ganado.

- El alimento es netamente orgánico, además de contener minerales en forma natural, necesarios para una dieta saludable.

- Los agricultores optimizan el uso de árboles, pasto permanente y otras especies perennes que secuestran carbono y reducen el efecto de los gases de tipo invernadero.

- Los agricultores optimizan el uso del abono, almacenamiento de lodo y método de aplicación y ritmo para prevenir las pérdidas de metano y óxido nitroso.

- Todas las operaciones trabajan para minimizar las emisiones de carbono de motores de combustión interna. Deberán convertirse en híbridas o alguna otra alternativa con menor emisión de gas propano líquido, metano, o agrocombustibles. 
- Los operadores eliminan el uso de todo clorofluorocarbono e hidrofluorocarbono en refrigerantes y otras aplicaciones.

- Todas las operaciones aspiran a aumentar la eficiencia de la energía y a reducir la dependencia en fuentes no renovables de energía a través de una mejora en las técnicas de manejo y tecnología.

- Las operaciones apoyan los cambios en infraestructura necesaria para aumentar el uso de fuentes renovables de energía administradas responsablemente. Se evita en lo posible el uso de energía proveniente de fuentes que dañan el entorno, ya sea a través de un impacto ambiental negativo de su infraestructura, $u$ otras características contaminantes.

\section{Dimensión Económica}

- El invertir en operaciones agrícolas incluye invertir en la salud.

- El costo real de producir alimentos sostenibles es compartido por toda la cadena de valor, para que todos los vínculos necesarios - especialmente los agricultores - puedan mantenerse en el negocio.

- Cualquier deuda es capitalizada para permitir que el operador cumpla con sus obligaciones actuales y previsibles.

- Las operaciones muestran preferencia en la selección de sus bancos para aquellos que tienen una clara política que reconoce la sostenibilidad.

- Los procesadores y/o comerciantes invierten recursos en capacitación y asistencia para conseguir cualquier permiso o certificación necesaria para tener acceso al mercado.

- Los compradores apoyan la economía rural a través de la mejora del dominio de fincas, prefiriendo situaciones agrícolas que apoyan el dominio de las mismas.

- Los agricultores aspiran a un balance en sus fuentes de ingresos para ayudar a asegurar la estabilidad de sus negocios.

- Procesadores y vendedores de productos sostenibles: o Suministran productos de alta calidad solicitados por los clientes; o Ejecutan estudios de mercado para entender mejor la demanda y los patrones de tendencia.

- La información básica de ventas y mercadeo incluye los siguientes aspectos, los cuales pueden ser encontrados en la etiqueta de un producto o de lo contrario, a través de una página web u otro tipo de material de mercadeo.
- Las fuentes de materiales no-renovables son importantes de considerar ya que extraerlos de la tierra puede tener un importante impacto ambiental.

- No se usan materiales que se sospeche contengan un alto nivel de contaminantes tales como residuos de insecticidas, transgénicos, o metales pesados. Proveedores y agricultores investigan la fuente de materiales para determinar cualquier posible amenaza de contaminación, y de acuerdo a eso guían sus acciones.

- Se reduce el uso de materiales de empaque. En orden de preferencia: se prefieren materiales reusados y luego reciclados, y éstos a su vez son reusables, reciclables y compostables.

\section{Dimensión Cultural}

- Los actores de la cadena de valor respetan la sabiduría indígena y los derechos a la propiedad intelectual, reconocen la fuente y valor del conocimiento cultural y tradicional, y las innovaciones tecnológicas, y compensan justamente a sus fuentes bajo términos de mutuo acuerdo.

- Los actores de la cadena de valor participan en debates de orden público y/o intentan influenciar el orden público en dirección de la sostenibilidad y el apoyo del desarrollo local cultural y de la comunidad, en la medida que sus posibilidades lo permitan.

- Los actores de la cadena de valor mejoran la calidad de vida de las personas y comunidades que producen los productos.

- En lugares donde la seguridad alimentaria es un problema, la empresa (agrícola u otro actor de la cadena de valor) contribuye a la seguridad alimentaria de su personal, sus familias, y la comunidad local.

- En regiones donde se ha reconocido que el hambre o la nutrición y el acceso a una alimentación adecuada es un problema, la prioridad de abastecer alimentos en el mercado se extiende desde los vecinos más cercanos geográficamente hacia fuera, de familia de finca a localidad o pueblo, a región, nación, y luego más allá.

- El procesamiento de alimentos es mantenido al mínimo y realizado de manera natural. Se usa la tecnología apropiada para ayudar a retener el valor nutricional de los alimentos y sus perfiles de sabor inherentes.

- Los alimentos procesados son formulados y fabricados en maneras que respetan la herencia cultural y minimizan la pérdida de valor nutricional. 


\section{Dimensión de Responsabilidad Social}

- Los límites del sistema están definidos para abarcar cualquier movimiento de material desde su origen hasta su disolución final o deposición.

- Los eslabones subsecuentes en la cadena de valor son responsables por conocer de antemano y hacer que los impactos cumulativos sean transparentes y que incluyan sus propias actividades. En particular, se debe prestar atención a la manera en que éstos impactos se relacionan con reclamos en cuanto a su nivel de sostenibilidad.

- Para cualquier sistema en revisión, los límites y alcance de los informes contables y financieros son claros, por ejemplo, lo cubierto vs. lo que está fuera del alcance de los informes. Si una organización no aborda todos los temas descritos en este documento, justifican sus omisiones.

- Los operadores escogen indicadores y parámetros que abordan el espectro total de los asuntos de sostenibilidad mencionados en este documento. • Los operadores comparan los valores iniciales de actividades con las medidas tomadas más adelante.

- Los participantes de la cadena de valor indemnizan por los impactos negativos en el medio ambiente al usar energía no-renovable, emisión de gases de efecto invernadero, emisión o liberación de toxinas en el medio ambiente durante cualquier etapa de producción de la cadena de valor, incluyendo el transporte y almacenamiento.

- Todas las partes interesadas sustancialmente afectadas por las actividades de la empresa son identificadas y luego habilitadas para compartir la toma de decisiones en actividades que impactan sus vidas y entorno.

- Antes de tomar decisiones con gran potencial de impactos de sostenibilidad, las empresas realizan las diligencias debidas y todas las partes interesadas pueden acceder fácilmente a los resultados.

- Los conflictos de intereses de las partes interesadas son resueltos a través de un apropiado diálogo directo o con mediación basada en el respeto, entendimiento mutuo, justa resolución de conflicto, y poder equitativo.

\section{CONCLUSIONES}

1. Se evidencia que la ineficiencia en los procesos productivos agrícolas es producto de aplicar prácticas contaminantes que causan daño a la tierra y recursos naturales, impactando el abastecimiento de alimentos y salud de la población.

2. Se establece el valor agregado de la agricultura orgánica en el sistema de producción sostenible, la que acompaña muchos beneficios como son económicos, sociales y ambientales.

3. Ha quedado establecido que un modelo de producción agrícola sostenible, permite reducir el impacto ambiental y el mejor uso de los recursos naturales, garantizando sostenibilidad en las actividades productivas y sustentabilidad alimenticia.

\section{RECOMENDACIONES}

1. El Estado debe difundir las mejores prácticas en sostenibilidad y sustentabilidad, a fin de lograr una cadena productiva sostenible en la agricultura orgánica y reivindicación del productor agrícola, causante del mal uso de los recursos naturales y daño al medio ambiente.

2. Los operadores de buenas prácticas en los procesos de producción orgánica, deben abordar diferentes dimensiones que consideren aspectos sociales, ecológicos, económicos, culturales y de responsabilidad social.

3. Los empresarios deben utilizar modelos de producción sostenible aplicando buenas prácticas para mitigar el impacto ambiental y optimizar los procesos y buen uso de los recursos naturales, garantizando la seguridad alimenticia y la salud de la población. 


\section{REFERENCIAS BIBLIOGRÁFICAS}

1. DE CAMINO, R.( 2005) El manejo de bosques y plantaciones forestales al 2025 en Costa Rica. ¿Qué podemos esperar? "El sector forestal en Costa Rica, perspectivas al 2025. Costa Rica.

2. FRONTI DE GARCIA, L (2009) Sumideros y mecanismo de desarrollo limpio: una propuesta de desarrollo sostenible para países del MERCOSUR, documentos de trabajo de Contabilidad Patrimonial y Ambiental.

3. SILVA, J (2011) Sistema de información ambiental y económica integrada. Ámbito internacional. ONU.

4. QUINTERO, M. (2006) Modelo de optimización para evaluación ex ante de alternativas productivas y cuantificación de externalidades ambientales en Cuencas Andinas. Proyecto Regional Cuencas Andinas CONDESAN y Centro Internacional de Agricultura Tropical CIAT, Cali, Colombia.

\section{HEMEROGRÁFICAS}

1. Autoridad Autónoma de Cuenca Hidrográfica Chira Piura y PDRS, GTZ(2004).Diagnóstico participativo con enfoque de gestión del riesgo de la Cuenca del Río Piura, Piura, Perú.

2. Banco Interamericano de Desarrollo-BID. (2005). Estudio sobre inversión directa en negocios forestales sostenibles - proyecto atn/ np-8323-r. Índice de atracción a la inversión forestal (IAIF) Informe Final - rev. 01. División de administración de recursos naturales y medio ambiente departamento regional de operaciones. Curitiba - Brasil. Abril / 2005.

3. GÓMEZ, R (2012) La agricultura orgánica: los beneficios de un sistema de producción sostenible. Centro de Investigación de la Universidad del Pacífico. Documento de Discusión DD/12/13

4. HELFGOTT, S., VARGAS, S., GUTIÉRREZ, A.

5. \& SALVATORE, M. (2010) Aptitud de tierras y oportunidades para el desarrollo rural en Perú.

6. Informe de la Comisión Mundial sobre el Medio Ambiente y el Desarrollo (Comisión Brundtland): Nuestro Futuro Común ONU (11/12/1987).

7. PIZARRO, N. (2010). Productos orgánicos.

8. Red de Acción de Agricultura Sostenible SOAAN (2013). Guía de mejores prácticas para la agricultura y cadena de valor. Versión pública, diciembre 2013.

\section{ELECTRÓNICAS}

1. IFOAM - Federación Internacional de Movimientos Agrícolas Orgánicos www.ifoam.org. visita 13-11-15.

2. http//www.monografía.com/trabajos87/cadenas-productivas-agrícolas-desarrollo-local-sostenible, Visita 4-12-14. 